\title{
Mineral and bone disorder biomarkers and inflammation indexes in patients with end stage renal disease
}

\author{
Lu Zhang ${ }^{1,2 \#}$, Qingtan Yu ${ }^{3 \#}$, Xing Chen ${ }^{1,2}$, Mengqin Zhang ${ }^{4}$, Yan $\mathrm{He}^{4}$, Zhiqian Ji ${ }^{4}$, Rongjuan Chen ${ }^{4}$, \\ Jun Zhang ${ }^{1,2}$, Hengyuan Zhang ${ }^{1,2}$, Guixiu Shi ${ }^{4}$, Jiyi Huang ${ }^{1,2}$
}

${ }^{1}$ Department of Nephrology, The First Affiliated Hospital of Xiamen University, Xiamen, China; ${ }^{2}$ The Fifth Hospital of Xiamen, Xiang'an Branch, The First Affiliated Hospital of Xiamen University, Xiamen, China; ${ }^{3}$ Department of laboratory, Qingdao Special Servicemen Recuperation Center of PLA Navy, Qingdao, China; ${ }^{4}$ Department of Rheumatology, The First Affiliated Hospital of Xiamen University, Xiamen, China

Contributions: (I) Conception and design: L Zhang, Q Yu, G Shi, J Huang; (II) Administrative support: X Chen, M Zhang; (III) Provision of study materials or patients: Y He, Z Ji; (IV) Collection and assembly of data: L Zhang, R Chen, Jun Zhang, Hengyuan Zhang; (V) Data analysis and interpretation: L Zhang, G Shi, J Huang; (VI) Manuscript writing: All authors; (VII) Final approval of manuscript: All authors.

\#These authors contributed equally to this work.

Correspondence to: Guixiu Shi. Department of Rheumatology, The First Affiliated Hospital of Xiamen University, Xiamen, China.

Email: gshi@xmu.edu.cn; Jiyi Huang. Department of Nephrology, The First Affiliated Hospital of Xiamen University, Xiamen, The Fifth Hospital of Xiamen, Xiang'an Branch, The First Affiliated Hospital of Xiamen University, Xiamen, China. Email: hjy0602@163.com.

Background: Chronic kidney disease (CKD) is an independent risk factor for bone and mineral metabolism disorder. Bone and mineral metabolism disorder develop gradually with the progression of renal failure. Various abnormalities include elevated fibroblast growth factor-23, decreased levels of 1,25-Dihydroxy Vitamin D (1,25-(OH)2D), and secondary hyperparathyroidism. This study aimed to evaluate the biomarkers and inflammation indexes of CKD-mineral and bone disorder (MBD), and to examine the associations of serum alkaline phosphatase (ALP) levels with serum C-reactive protein (CRP) levels and leukocyte count, in patients with end-stage renal disease (ESRD).

Methods: ESRD patients who were yet to initiate dialysis were enrolled as the study subjects. All CKD patients who were discharged from our hospital between January 1, 2013, and December 31, 2017, were screened. Based on quartiles of serum ALP, all subjects were categorized into four subgroups. The clinical characteristics of patients in the four subgroups were investigated. The prevalence of hypocalcemia, hyperphosphatemia, elevated intact parathyroid hormone (iPTH), and elevated CRP level was calculated in the four subgroups. The association between serum ALP and inflammation indexes was examined using regression analysis.

Results: Based on the inclusion criteria and exclusion criteria, 256 cases with ESRD were included in our study. The overall prevalence of hypocalcemia and hyperphosphatemia was $57.81 \%$ and $89.84 \%$, respectively. Of the patients with ESRD, 174 (68.75\%) had an elevated iPTH level $>2$ times of the upper limit of normal value), and 40 (15.63\%) had an elevated iPTH level >9 times of the upper limit of normal value Discrepancies between serum ALP and iPTH levels were found in 29 patients. In the adjusted model, log-transformed CRP, log-transformed iPTH, and leukocyte count were significantly associated with logtransformed ALP.

Conclusions: Hypocalcemia, hyperphosphatemia, and increased levels of iPTH and CRP were found to be extremely common in ESRD patients. Discrepancies between serum ALP and iPTH were also observed. Our results also indicate that serum ALP level is associated with the levels of iPTH and CRP, as well as leukocyte count.

Keywords: Bone biomarkers; inflammation indexes; kidney disease

Submitted Aug 24, 2020. Accepted for publication Nov 18, 2020.

doi: 10.21037/apm-20-2083

View this article at: http://dx.doi.org/10.21037/apm-20-2083 


\section{Introduction}

Chronic kidney disease (CKD) is an independent risk factor for bone and mineral metabolism disorder $(1,2)$. As renal failure progresses, bone and mineral metabolism disorder gradually develops. Patients with CKD exhibit various abnormalities, including elevated levels of fibroblast growth factor-23 (FGF23), decreased levels of 1,25-Dihydroxy Vitamin D [1,25-(OH)2D], and secondary hyperparathyroidism $(1,2)$. Overt hyperphosphatemia may occur even if estimated glomerular filtration rate (eGFR) is $>30 \mathrm{~mL} / \mathrm{min}$ per $1.73 \mathrm{~m}^{2}$, however, it is not common (3). $\mathrm{CKD}$ is also associated with multiple bone disorders, such as osteitis fibrosa (4), osteomalacia (4), adynamic osteopathy $(4,5)$ and mixed uremic osteodystrophy (4). Treatment of CKD-mineral and bone disorder (CKD-MBD) included lowering phosphate and calcium maintenance, treatment of hyperparathyroidism, treatment of bone abnormalities (6).

In recent years, the embryonic origin of bone cells has been demonstrated to confer the potential for systemic extra-skeletal effects on bone $(7,8)$. Bone is currently recognized as an endocrine organ $(7,8)$, and bone cells produce osteocalcin (OC), FGF23, and sclerostin (Sost) $(7,8)$. In end-stage renal disease (ESRD), CKD-MBD contributes to cardiovascular events and poor outcomes $(1,7,8)$. CKD-MBD has become an urgent global public health issue (9). Thus, recognizing the specific types of renal osteodystrophy is of increasing importance $(1,2,7,8)$. Despite transiliac bone biopsy being reliable for observing histomorphometric changes and cellular activity in bone tissue (10-12), it is not routinely used for inspection in clinical practice. Laboratory biomarkers are being continuously developed and can provide relatively accurate information $(1,2,3,8,13,14)$. Chronic inflammation has increasingly been recognized as a feature of CKD (8), and a link between inflammation and bone has also been established (15). During the process of bone injury repair, bone cells and inflammatory cells are both recruited, resulting in intense crosstalk (15). Chronic inflammation can also affect the activity of bone cells (8).

Alkaline phosphatases (ALP) are responsible for removing phosphate $(\mathrm{P})$, which is involved not only in bone mineralization but also in vascular calcification $(1,2,14)$. ALP is involved in cross-talk between bone and vessels $(2,14)$. In CKD, total ALP is generally regarded as a reliable marker of bone turnover $(1,2,14)$. An association between serum ALP and elevated serum C-reactive protein (CRP) levels has been reported in CKD patients (16) and
nor-CKD population $(17,18)$. Although various studies of the biomarkers of CKD-MBD have been performed in ESRD patients, a majority of the previous studies involved patients undergoing dialysis. The conventional dialysate is phosphorus free and a relatively high-calcium caution resolution.

Dialysis can effectively improve disorders of calcium and phosphorus metabolism (1). CKD-MBD is an intricate network $(1,2)$. A change to one component could potentially affect the whole network. Dialysis patients may not be the ideal subjects for presenting the natural course of CKDMBD in patients ESRD. The present study was performed to address the current lack of relevant research on this topic. Therefore, with ESRD patients who were yet to initiate dialysis and CKD-MBD-targeted treatment selected as the study subjects, we aimed to evaluate the CKD-MBD biomarkers and inflammation indexes, and to examine the associations of serum ALP levels with serum CRP levels and leukocyte counts in these patients. We present the following article in accordance with the STROBE reporting checklist (available at http://dx.doi.org/10.21037/apm-20-2083).

\section{Ethics statement}

Before starting the present study, we submitted our study protocol for ethical approval. The study was approved by Ethics Committee The First Affiliated Hospital of Xiamen University (2020033). The study was conducted in strict accordance with the Declaration of Helsinki of the World Medical Association (as revise in 2013). Need for written informed consent from the patients also has been waived by hospital ethics committee.

\section{Methods}

\section{Patients}

ESRD patients who were yet to initiate dialysis were selected as our study subjects. All CKD patients who were discharged from our hospital between January 1, 2013, and December 31, 2017, were screened. For inclusion in the study, subjects needed to meet all of the following criteria: (I) diagnosed with ESRD, which was defined as eGFR $<15 \mathrm{~mL} / \mathrm{min} / 1.73 \mathrm{~m}^{2}$ and renal disease $>90$ days; (II) not yet on dialysis; (III) had not received targeted treatment for CKD-MBD, including phosphorus binders, vitamin D3, calcitriol, calcitriol analogue, and calcium mimetic (1). Patients with missing data on CKD-MBD biomarkers were 
excluded from the study.

\section{CKD-MBD biomarkers and inflammation indexes}

CKD-MBD biomarkers including serum total calcium, serum phosphorus, serum calcium phosphorus product, intact parathyroid hormone, and serum total ALP were analyzed in this study. Serum total calcium was determined using the Arsenazo III method (Calcium Assay Kit by Arsenazo III Method \#CH0101251R1, Maccura biotechnology Co, China); Serum phosphorus was determined by using UV Method method (Inorganic Phosphorus Assay Kit \#CH0101253R1, Maccura biotechnology co, china); and serum creatinine was determined using the ammonia oxidase method (Creatinine Assay Kit by SAO Method, \#CH0101053, Maccura biotechnology Co, China). Total ALP and intact PTH were measured using the NPP-substrate AMP-buffer method and the point electroluminescence method (Siemens immulite 1000 systems iPT, \#LKPP1, Siemens Healthcare Diagnostics Products Ltd, UK), respectively. In the present study, high-sensitivity CRP determined by rate nephelometry (Creactive Protein Assay Kit by Immunoturbidimetry Method, \#CH0181303 maccura biotechnology Co, China) was used as a biomarker for chronic inflammation index. Leukocyte count results were extracted from hospital record detected by Miradray blood analyzer. Because ferritin is also associated with chronic inflammation (16), it was also included in our data analysis. The recommended normal reference values of the laboratory indicators were as follows: serum total calcium, 2.08-2.60 mmol/L; serum phosphorus, $1.00-1.60 \mathrm{mmol} / \mathrm{L}$; serum creatinine, 44-106 $\mu \mathrm{mol} / \mathrm{L}$; serum total ALP, $35.0-135.0 \mathrm{U} / \mathrm{L}$; and iPTH, $15-65 \mathrm{pg} / \mathrm{mL}$. In the present study, hypocalcemia was defined as a serum calcium level $<2.08 \mathrm{mmol} / \mathrm{L}$. Hyperphosphatemia was defined as a serum phosphorus level $>1.60 \mathrm{mmol} / \mathrm{L}$. Elevated hsCRP was defined as hsCRP $>3.0 \mathrm{mg} / \mathrm{L}$, and severe elevated hsCRP was defined as hsCRP $>10.0 \mathrm{mg} / \mathrm{L}$. iPTH was divided into three levels: $<2$ times of the upper limit of normal value $(<130 \mathrm{pg} / \mathrm{mL}), 2-9$ times of the upper limit of normal value $(130-585 \mathrm{pg} / \mathrm{mL})$, and 9 times of the upper limit of normal value $(>585 \mathrm{pg} / \mathrm{mL})$. Because a previous study indicated an association of serum ALP and leukocyte count (15), we also included leukocyte count in our statistical analysis.

GFR was estimated according to the Modification of Diet in Renal Disease (MDRD) equation $[175 \times(\mathrm{Scr} \mathrm{mg} / \mathrm{dL})$ $-1.234 \times$ (age) $-0.179 \times($ if female, $\times 0.79)$ ] (19).
Discrepancies between serum ALP and iPTH reflected an uncoupling between bone resorption and formation $(14,20)$. Although they are uncommon in the general population, discrepancies between serum ALP and iPTH have been reported in dialysis patients $(14,20,21)$. In the present study, a discrepancy between serum ALP and iPTH was defined in the following two scenarios: high serum ALP $(>80.0 \mathrm{U} / \mathrm{L})$ and a low level of PTH $(<150 \mathrm{pg} / \mathrm{mL})$, or low serum ALP $(<35 \mathrm{U} / \mathrm{L})$ and a high level of PTH $(>200 \mathrm{pg} / \mathrm{mL})$.

\section{Collection of clinical parameters}

The patients' medical records were retrospectively reviewed. Patient demographic information, medical history, and laboratory data were obtained. The average value of three blood pressure measurements taken after admission was calculated and used as blood pressure. Infection was defined as a diagnosis of infection indicated in the medical records and treated with antibiotics.

\section{Statistical analysis}

Data were analyzed using Stata (version 14.0) statistical software. Continuous variables with normal distribution were presented as mean $\pm \mathrm{SD}$, and those with skewed distribution were presented as median and quartile. Categorical data were presented as absolute and percentage. Clinical characteristics of subjects were presented.

According to quartiles of serum ALP, all subjects were categorized into four subgroups: ALP Q1: 0-25\%, Q2: 25-50\%, Q3: 50-75\% and Q4 >75\%. The clinical characteristics of the four subgroups are presented in a table. Differences in multiple sets of data were compared by one-way analysis of variance or the Kolmogorov-Smirnov test according to the characteristics of the variables. A Bonferroni test was used for multiple comparisons. The chisquare test was used to compare categorical variables. CRP, ALP, and PTH had skewed distribution in the CKD groups. CRP, ALP, and PTH were logarithmically transformed and then analyzed. Coefficients between log-transformed ALP and log-transformed PTH, log-transformed CRP, leukocyte count. and ferritin were analyzed using Pearson's correlation analysis. The models were adjusted for age, sex, body mass index (BMI), smoking, systolic blood pressure, and infection. A two-tailed $\mathrm{P}$ value $<0.05$ was considered to be statistically significant.

The prevalence of hypocalcemia, hyperphosphatemia, elevated iPTH level, and elevated CRP level were calculated 
Table 1 Clinical data of 256 end-stage renal disease (ESRD) patients

\begin{tabular}{|c|c|}
\hline Characters & Values $(\mathrm{N}=256)$ \\
\hline Age (years) & $56.32 \pm 14.95$ \\
\hline Male/female & $162 / 94$ \\
\hline $\mathrm{BMI}\left(\mathrm{kg} / \mathrm{cm}^{2}\right)$ & $22.89 \pm 3.27$ \\
\hline Systolic blood pressure (mmHg) & $157.58 \pm 25.95$ \\
\hline Diastolic blood pressure $(\mathrm{mmHg})$ & $91.05 \pm 14.70$ \\
\hline Smoker & 58 \\
\hline Diabetes & 60 \\
\hline \multicolumn{2}{|l|}{ Primary disease } \\
\hline Glomerulonephritis & 62 \\
\hline Diabetic nephropathy & 58 \\
\hline 24-hour urine volume (mL) & $1,100(810-1,500)$ \\
\hline Previous cardiovascular disease & 29 \\
\hline \multicolumn{2}{|l|}{ Biomarkers of CKD-MBD } \\
\hline Total serum calcium (mmol/L) & $1.96 \pm 0.37$ \\
\hline Phosphate (mmol/L) & $2.71 \pm 1.00$ \\
\hline Calcium phosphorus product (mmol/L) & $5.16 \pm 1.77$ \\
\hline PTH (pg/mL) & $303.4(178.05-452.3)$ \\
\hline Alkaline phosphatase (U/L) & $78.15(61.35-102)$ \\
\hline \multicolumn{2}{|l|}{ Other laboratory variables } \\
\hline Serum creatine $(\mu \mathrm{mol} / \mathrm{L})$ & $1054 \pm 406.12$ \\
\hline Urea (mmol/L) & $38.63 \pm 14.79$ \\
\hline Uric acid (mmol/L) & $598.06 \pm 157.21$ \\
\hline Leukocyte $\left(\times 10^{9}\right)$ & $8.04 \pm 4.46$ \\
\hline Proportion of neutrophils (\%) & $72.72 \pm 11.73$ \\
\hline Hemoglobin (g/L) & $77.62 \pm 20.46$ \\
\hline Platelets $\left(\times 10^{9}\right)$ & $193.07 \pm 76.75$ \\
\hline Albumin (U/L) & $34.53 \pm 5.42$ \\
\hline Prealbumin $(g / L)$ & $363.31 \pm 196.62$ \\
\hline Alanine aminotransferase (U/L) & $17.85(12.25-27.85)$ \\
\hline Glutamic oxaloacetate (U/L) & $19.9(15.5-27.0)$ \\
\hline Cholesterol (mmol/L) & $4.52 \pm 1.64$ \\
\hline Triglyceride (mmol/L) & $1.31(0.98-1.83)$ \\
\hline Low-density lipoprotein (mmol/L) & $2.63 \pm 1.11$ \\
\hline High-density lipoprotein (mmol/L) & $0.89 \pm 0.52$ \\
\hline C-reactive protein (g/L) & $5.83(1.44-22.40)$ \\
\hline
\end{tabular}

Table 1 (continued)
Table 1 (continued)

\begin{tabular}{lc}
\hline Characters & Values $(\mathrm{N}=256)$ \\
\hline Ferritin & $289.15(131.4-442.7)$ \\
Serum iron & $11.25(6.9-16.9)$ \\
Iron saturation & $29(17-42)$ \\
Total iron binding capacity (TIBC) & $43.29 \pm 11.85$ \\
Infection & 76 \\
Pulmonary infection & 57 \\
Urinary tract infection & 3 \\
Others & 5 \\
Multiple infections & 11 \\
\hline
\end{tabular}

BMI, body mass index; PTH, parathyroid hormone; CKD, chronic kidney disease; MBD, mineral and bone disorder.

in all subjects and four subgroups.

\section{Results}

All CKD patients who were discharged from our hospital between January 1, 2013, and December 31, 2017, were screened. Based on the inclusion criteria and exclusion criteria, 256 patients with ESRD were included in our study.

\section{Clinical data of 256 ESRD patients (Table 1)}

The detailed clinical data of 256 ESRD patients are displayed in Table 1. The patients had an average age of 56 years; 162 were male and 94 were female. As excepted, the ESRD patients had a lower level of total serum calcium, and a higher level of serum phosphorus and iPTH. The median serum ALP level was 78.15 (25\%: 61.35, $75 \%: 102)$. The inflammation indexes and the levels of CRP and ferritin were also elevated.

\section{Clinical data of ESRD patients according to serum ALP quartiles (Table 2)}

Based on quartiles of serum ALP, all subjects were categorized into four subgroups. The clinical characteristics of four subgroups are presented in Table 2. Data analysis showed significant differences in BMI, age, iPTH level, and ferritin level. No significant differences were found in the levels of total serum calcium, phosphorus, leukocytes, and 
Table 2 Clinical data of ESRD patients according to serum ALP quartiles

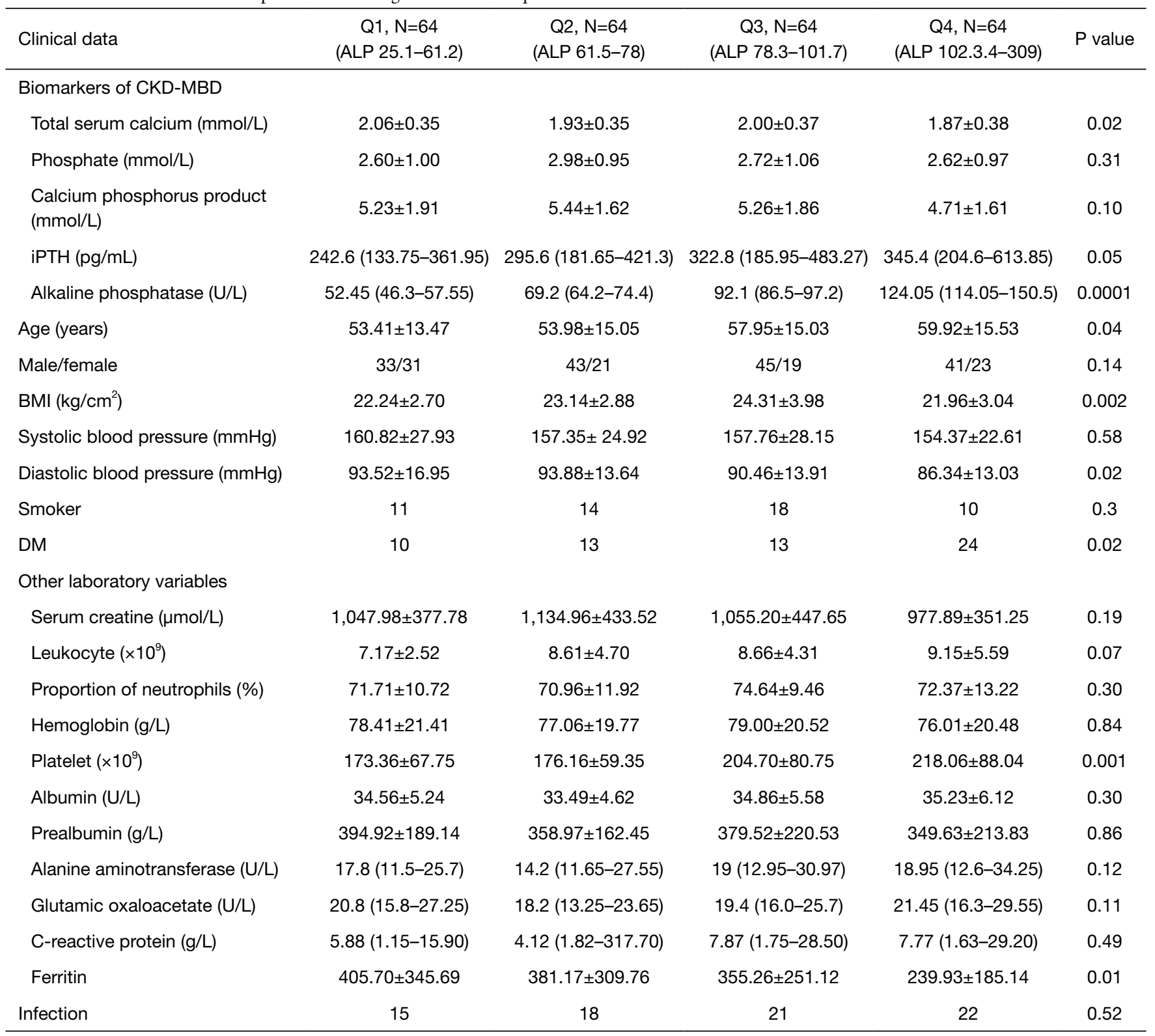

ESRD, end-stage renal disease; ALP, alkaline phosphatase; CKD, chronic kidney disease; MBD, mineral and bone disorder; iPTH, intact parathyroid hormone; BMI, body mass index; DM, diabetes mellitus.

CRP between the four subgroups.

\section{The prevalence on CKD-MBD biomarkers disorders and inflammation indexes according to serum ALP quartiles} (Table 3)

The overall prevalence of hypocalcemia and hyperphosphatemia was $57.81 \%$ and $89.84 \%$, respectively.
Of the 256 ESRD patients, 176 (68.75\%) had an elevated iPTH level $>2$ times of the upper limit of normal value and $40(15.63 \%)$ had an elevated iPTH level $>9$ times $t$ of the upper limit of normal value. Discrepancies between the levels of serum ALP and iPTH were found in 29 patients. Three patients had an elevated level of iPTH $(>200 \mathrm{pg} / \mathrm{mL})$ and a low level of ALP $(<35 \mathrm{U} / \mathrm{L})$, while 26 patients had a relatively higher level of ALP ( $>80 \mathrm{U} / \mathrm{L})$ and a low level of 
Table 3 Prevalence on CKD-MBD biomarker disorders and inflammation indexes according to serum ALP quartiles

\begin{tabular}{|c|c|c|c|c|c|c|}
\hline Biomarkers of CKD-MBD & Total, $\mathrm{N}=256$ & $\begin{array}{c}\mathrm{Q} 1, \mathrm{~N}=64 \\
(\mathrm{ALP} 25.1-61.2)\end{array}$ & $\begin{array}{c}\text { Q2, N=64 } \\
(\text { ALP 61.5-78) }\end{array}$ & $\begin{array}{c}\text { Q3, N=64 } \\
(\text { ALP 78.3-101.7) }\end{array}$ & $\begin{array}{c}\text { Q4, N=64 } \\
(\text { ALP 102.3.4-309) }\end{array}$ & $P$ value \\
\hline Hypocalcemia & $148(57.81 \%)$ & $32(50 \%)$ & $39(60.94 \%)$ & 35 (54.69\%) & $42(65.63 \%)$ & 0.29 \\
\hline PTH & & & & & & $<0.001$ \\
\hline CRP & & & & & & 0.97 \\
\hline CRP 3-10 & $35(13.67 \%)$ & 10 & 9 & 8 & 8 & \\
\hline$>10$ & $164(64.06 \%)$ & 39 & 39 & 42 & 44 & \\
\hline
\end{tabular}

CKD, chronic kidney disease; MBD, mineral and bone disorder. CRP, C-reaction protein; ALP, alkaline phosphatase; PTH, parathyroid hormone.

Table 4 Correlation of log-transformed ALP with log-transformed CRP, log-transformed PTH, leukocyte count, and ferritin

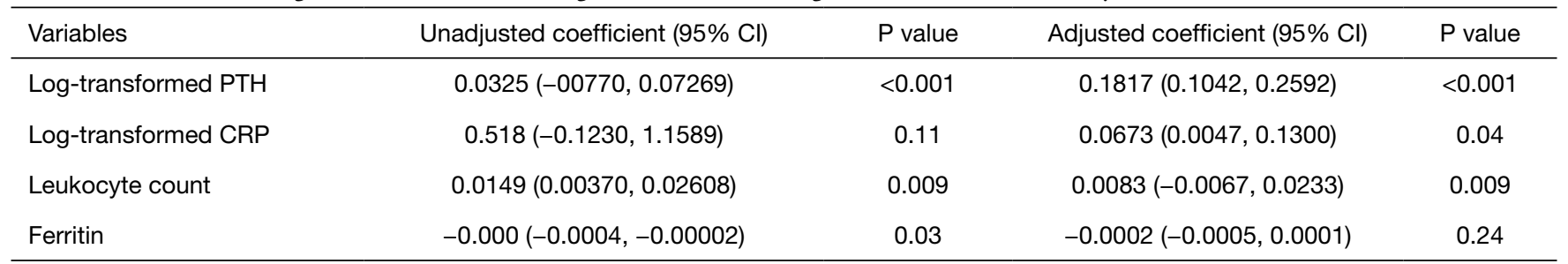

ALP, alkaline phosphatase; CRP, C-reaction protein; PTH, parathyroid hormone.

PTH $(<150 \mathrm{pg} / \mathrm{mL})$. A higher level of ALP may be related to elevated iPTH. However, no differences were observed in the prevalence of hypocalcemia, hyperphosphatemia, or elevated CRP between the four subgroups.

\section{Correlation of ALP with CRP level, leukocyte count, and ferritin (Table 4)}

The correlations of log-transformed ALP with logtransformed CRP, log-transformed PTH, leukocyte count, and ferritin were examined by adjusted regression models. The adjusted model showed log-transformed CRP, logtransformed PTH, and leukocyte count to be significantly associated with log-transformed ALP.

\section{Discussion}

In our study, we investigated CKD-MBD biomarkers and inflammatory indexes in ESRD patients who were yet to initiate dialysis. Unsurprisingly, hypocalcemia, hyperphosphatemia, and increased PTH and CRP were extremely common in ESRD patients. However, only 24 of the patients in our study were found to have an elevated ALP (>135 U/L). Discrepancies between serum ALP and iPTH were only recorded in 29 patients. After adjusting for age, sex, BMI, smoking, systolic blood pressure, and infection, serum ALP level was found to be associated with the level of iPTH and serum CRP level, and leukocyte count.

A number of studies have clarified that CKD attribute to bone and mineral disorders $(1,2)$. As renal failure progresses (CKD stage 3-4), the first change is typically early and progressive FGF23 elevation $(2,3)$. Then, calcitriol declines and parathyroid hormone increases to maintain phosphate levels within the normal range $(2,3)$. Usually, $70-80 \%$ of daily dietary phosphate intake is excreted in urine $(2,22)$. With further development of renal failure, eGFR decreases to $<30 \mathrm{~mL} / \mathrm{min} / 1.73 \mathrm{~m}^{2}$, and the level of phosphorus in the blood gradually increases (2). Data from the Dialysis Outcomes and Practice Patterns Study (DOPPS) for China (23) showed that severe hyperphosphatemia $(>7 \mathrm{mg} / \mathrm{dL})$ and 
secondary hyperparathyroidism (>600 pg/mL) were common ( $27 \%$ and $21 \%$, respectively) in patients undergoing hemodialysis. Decreased serum calcium levels $(<8.4 \mathrm{mg} / \mathrm{dL})$ were found in $25 \%$ patients on hemodialysis (21). Compared to other DOPPS regions [2012-2015], patients in these 45 Chinese dialysis units had a higher level PTH and phosphate (21). Unlike previous studies, our study focused on untreated CKD-MBD patients who had not yet received dialysis treatment. Symptoms of kidney diseases may be concealed. With the adaptation of chronic diseases, patients may ignore some symptoms, which may lead to a delay in the diagnosis of uremia. Even in the DOPPS study (23), CKD-MBD biomarkers were measured less frequently in China than in other DOPPS regions, with only $14.9 \%$ of Chinese patients undergoing frequent measurement for calcium and PTH (23). Phosphate binders were prescribed in $59 \%$ of Chinese patients (23). The data indicated that progress needed to be made in the management of CKDMBD. So, in the retrospective study, we noted a certain number of patients who had not received targeted treatment for CKD-MBD before undergoing hemodialysis. Since the completion of the DOPPS5 study, progress has been made with CKD-MBD management in China (23), and we have reason to believe that the diagnosis and treatment of CKDMBD can be further improved.

Transiliac bone biopsy is the gold standard for the diagnosis of renal osteopathy (1-3). However, it is not widely developed, and more often, biomarkers are used to assess bone turnover (1-3). Parathyroid hormone plays an important role in the regulation of bone turnover. However, the detection methods of iPTH are not accurate enough $(24,25)$. In the previous study (25), 10 detection methods were used to determine iPTH. The results of these different detection methods varied (25). In research on the relationship between iPTH and mortality in CKD patients, the optimal value of PTH recommended in different studies has also ranged widely (26). In view of this, the KDIGO guidelines recommend that the appropriate PTH value for dialysis patients is 2-9 times the normal value (26).

Total ALP is generally regarded as a reliable biomarker of bone turnover in CKD $(1,14,21)$. Circulating ALP is an independent predictor of all-cause mortality in the general population (27) as well as in CKD patients $(28,29)$. ALP is also involved in vascular calcification (30) and is a superior biomarker to iPTH for bone turnover in CKD patients $(14,21)$. The results of our study showed that an elevated iPTH was extremely common in ESRD patients who had not initiated dialysis. However, the median level of iPTH was lower than that in DOPPS [2012-2015] in China (23). Compared to the data on patients undergoing hemodialysis, we consider that hypocalcemia and hyperphosphatemia can be effectively corrected by dialysis and drug therapy; parathyroidism, however, is more difficult to deal with. Moreover, almost half of patients in the group had a relatively high level of ALP (>80 U/L). A combination of ALP and iPTH is an effective tool for predicting bone turnover $(14,21,24)$. Discrepancies between serum iPTH and BSAP levels may be found in CKD patients $(21,24)$. One explanation for this is the variability in the measurements of ALP and iPTH $(21,24)$. Increased serum iPTH levels with low ALP may reflect skeletal resistance or decreased calcemic response to PTH (19,30-33). In our study, only three patients had elevated iPTH (>200 pg/mL) with a low level of ALP ( $<35 \mathrm{U} / \mathrm{L})$.

A potential explanation for the association of serum ALP with mortality in the CKD population is inflammation (16). The association of CRP and ALP has been found in the general population as well as CKD patients (34). Using NHANES III with valid data, analysis indicated that serum ALP was independently associated with elevated CRP in the CKD and non-CKD populations (34). In our study, in the unadjusted model, the association of CRP and ALP was not significant. However, after adjusting for potential confounding factors, ALP was found to be associated with CRP. Similar to a previous study based on a Korean population (17), we also find that ALP level was independently associated with leukocyte count after controlling for confounding factors. An association between elevated ALP and inflammatory markers may provide a reasonable explanation for the association of ALP and mortality.

Total ALP includes tissue and non-tissue specific alkaline (TNAP). TNAPs are present in several tissues, including the liver, the kidney, and bone (21); those found in the bone are referred to as bone-specific APs (BSAPs) (19). BSAPs are a very specific bone formation marker. Although ALP is regarded as a reliable marker of bone turnover in CKD $(1,14,21)$, elevated ALP level can also be caused by liver damage. Moreover, intestinal AP may also be increased in hemodialysis patients. BSAPs are also more sensitive and specific for bone disease. In our study, ALP was used as the biomarker of CKD-MBD rather than BSAPs, which is a limitation of our study. Other limitations include our study's retrospective nature. 


\section{Conclusions}

As expected, hypocalcemia, hyperphosphatemia, and increased PTH and CRP levels were extremely common in the ESRD patients in our study. Discrepancies between serum ALP and iPTH were also found these patients. Our results also indicate that serum ALP level is associated with the levels of iPTH and CRP, as well as leukocyte count.

\section{Acknowledgments}

Funding: LZ is supported by the National Natural Science Foundation of China (Grant No. 81900657) and Natural Science Foundation of Fujian Province (Grant No.2020J011243).

\section{Footnote}

Reporting Checklist: The authors have completed the STROBE reporting checklist. Available at http://dx.doi. org/10.21037/apm-20-2083

Data Sharing Statement: Available at http://dx.doi. org/10.21037/apm-20-2083

Conflicts of Interest: All authors have completed the ICMJE uniform disclosure form (available at http://dx.doi. org/10.21037/apm-20-2083). The authors have no conflicts of interest to declare.

Ethical Statement: The authors are accountable for all aspects of the work in ensuring that questions related to the accuracy or integrity of any part of the work are appropriately investigated and resolved. The study was approved by Ethics Committee The First Affiliated Hospital of Xiamen University (2020033). The study was conducted in strict accordance with the Declaration of Helsinki of the World Medical Association (as revise in 2013). Need for written informed consent from the patients also has been waived by hospital ethics committee.

Open Access Statement: This is an Open Access article distributed in accordance with the Creative Commons Attribution-NonCommercial-NoDerivs 4.0 International License (CC BY-NC-ND 4.0), which permits the noncommercial replication and distribution of the article with the strict proviso that no changes or edits are made and the original work is properly cited (including links to both the formal publication through the relevant DOI and the license). See: https://creativecommons.org/licenses/by-nc-nd/4.0/.

\section{References}

1. Kidney Disease: Improving Global Outcomes (KDIGO) CKD-MBD Update Work Group. KDIGO 2017 Clinical Practice Guideline Update for the Diagnosis, Evaluation, Prevention, and Treatment of Chronic Kidney DiseaseMineral and Bone Disorder (CKD-MBD). Kidney Int Suppl 2017;7:1-59.

2. Isakova T, Ix JH, Sprague SM, et al. Rationale and Approaches to Phosphate and Fibroblast Growth Factor 23 Reduction in CKD. J Am Soc Nephrol 2015;26:2328-39.

3. Isakova T, Wahl P, Vargas GS, et al. Fibroblast growth factor 23 is elevated before parathyroid hormone and phosphate in chronic kidney disease. Kidney Int 2011;79:1370-8.

4. Sherrard DJ, Hercz G, Pe Y, et al. The Spectrum of Bone Disease in End-Stage Renal Failure--An Evolving Disorder. Kidney Int 1993;43:436-42.

5. Malluche HH, Monier-Faugere MC. Risk of adynamic bone disease in dialyzed patients. Kidney Int Suppl 1992;38:S62-7.

6. KettelerM, Block GA, Evenepoel P, et al. Executive summary of the 2017 KDIGO Chronic Kidney DiseaseMineral and Bone Disorder (CKD-MBD) Guideline Update: what's changed and why it matters. Kidney Int 2017;92:26-36.

7. Vervloet M, Massy Z, Brandenburg V, et al. Bone: a new endocrine organ at the heart of chronic kidney disease and mineral and bone disorders. Lancet Diabetes Endocrinol 2014;2:427-36.

8. Bazzaferro S, Cianciolo G, De Pascalis A, et al. Bone, inflammation and the bone marrow niche in chronic kidney disease: what do we know? Nephrol Dial Transplant 2018;33:2092-100.

9. Nizet A, Cavalier E, Stenvinkel P, et al. Bone alkaline phosphatase: An important biomarker in chronic kidney disease - mineral and bone disorder. Clin Chim Acta 2020;501:198-206.

10. e Vernejoul MC, Kuntz D, Miravet L, et al. Bone histomorphometry in hemodialysed patients. Metab Bone Dis Relat Res 1981;3:175-9.

11. Keronen S, Martola L, Finne P. Bone Histomorphometry and Indicators of Bone and Mineral Metabolism in WaitListed Dialysis Patients. Clin Nephrol 2016;85:127-34.

12. Ramalho J, Marques IDB, Hans D, et al. The trabecular 
bone score: Relationships with trabecular and cortical microarchitecture measured by HR-pQCT and histomorphometry in patients with chronic kidney disease. Bone 2018;116:215-20.

13. Delanaye P, Souberbielle JC, Lafage-Proust MH, et al. Can we use circulating biomarkers to monitor bone turnover in CKD haemodialysis patients? Hypotheses and facts. Nephrol Dial Transplant 2014;29:997-1004.

14. Haarhaus M, Brandenburg V, Kalantar-Zadeh K, et al. Alkaline phosphatase: a novel treatment target for cardiovascular disease in CKD. Nat Rev Nephrol 2017;13:429-442

15. Loi F, Córdova LA, Pajarinen J, et al. Inflammation, fracture and bone repair. Bone 2016;66:119-30.

16. Damera S, Raphael KL, Baird BC, et al. Serum alkaline phosphatase levels associate with elevated serum C-reactive protein in chronic kidney disease. Kidney Int 2011;79:228-33.

17. Seo MS, Shim JY, Lee YJ. Relationship between serum alkaline phosphatase level, C-reactive protein and leukocyte counts in adults aged 60 years or older. Scand J Clin Lab Invest 2019;79:233-7.

18. Rambod M, Kovesdy CP, Kalantar-Zadeh K. Combined High Serum Ferritin and Low Iron Saturation in Hemodialysis Patients: The Role of Inflammation. Clin J Am Soc Nephrol 2008;3:1691-701.

19. Ma YC, Zuo L, Chen JH, et al. Modified glomerular filtration rate estimating equation for Chinese patients with chronic kidney disease. J Am Soc Nephrol 2006;17:2937-44.

20. Chen Z, Zhang X, Han F, et al. High Alkaline Phosphatase and Low Intact Parathyroid Hormone Associate With Worse Clinical Outcome in Peritoneal Dialysis Patients. Perit Dial Int 2020;2020:896860820918131.

21. Bover J, Ureña P, Aguilar A, et al. Alkaline Phosphatases in the Complex Chronic Kidney Disease-Mineral and Bone Disorders. Calcif Tissue Int 2018;103:111-24.

22. Blaine J, Chonchol M, Levi M. Renal Control of Calcium, Phosphate, and Magnesium Homeostasis. Clin J Am Soc Nephrol 2015;10:1257-72.

23. Wang J, Bieber BA, Hou FF, et al. Mineral and Bone Disorder and Management in the China Dialysis Outcomes and Practice Patterns Study. Chin Med J (Engl) 2019;132:2775-82.

24. Soliman M, Hassan W, Yaseen M, et al. PTH Assays in Dialysis Patients: Practical Considerations. Semin Dial 2019;32:9-14.

25. Cavalier E, Delanaye P, Vranken L, et al. Interpretation of serum PTH concentrations with different kits in dialysis patients according to the KDIGO guidelines: importance of the reference (normal) values. Nephrol Dial Transplant 2012;27:1950-6.

26. Kidney Disease: Improving Global Outcomes (KDIGO) CKD-MBD Work Group. KDIGO clinical practice guideline for the diagnosis, evaluation, prevention, and treatment of Chronic Kidney Disease-Mineral and Bone Disorder (CKD-MBD). Kidney Int Suppl 2009;(113):S1-130.

27. Kunutsor SK, Apekey TA, Khan H. Liver enzymes and risk of cardiovascular disease in the general population: a meta-analysis of prospective cohort studies. Atherosclerosis 2014;236:7-17.

28. Liu X, Guo Q, Feng X, et al. Alkaline Phosphatase and Mortality in Patients on Peritoneal Dialysis. Clin J Am Soc Nephrol 2014;9:771-8.

29. Fan Y, Jin X, Jiang M, et al. Elevated Serum Alkaline Phosphatase and Cardiovascular or All-Cause Mortality Risk in Dialysis Patients: A Meta-Analysis. Sci Rep 2017;7:13224.

30. Azpiazu D, Gonzalo S, Villa-Bellosta R. Tissue NonSpecific Alkaline Phosphatase and Vascular Calcification: A Potential Therapeutic Target. Curr Cardiol Rev 2019;15:91-5.

31. Evenepoel P, Bover J, Ureña Torres P. Parathyroid hormone metabolism and signaling in health and chronic kidney disease. Kidney Int 2016;90:1184-90.

32. Fukagawa M, Kazama JJ, Shigematsu T. Skeletal Resistance to PTH as a Basic Abnormality Underlying Uremic Bone Diseases. Am J Kidney Dis 2001;38:S152-5.

33. Llach F, Massry S, Singer F, et al. Skeletal resistance to endogenous parathyroid hormone in patients with early renal failure. A possible cause for secondary hyperparathyroidism. J Clin Endocrinol Metab 1975;41:339-45.

34. Filipowicz R, Greene T, Wei G, et al. Associations of serum skeletal alkaline phosphatase with elevated C-reactive protein and mortality. Clin J Am Soc Nephrol 2013;8:26-32.

Cite this article as: Zhang L, Yu Q, Chen X, Zhang M, He Y, Ji Z, Chen R, Zhang J, Zhang H, Shi G, Huang J. Mineral and bone disorder biomarkers and inflammation indexes in patients with end stage renal disease. Ann Palliat Med 2020;9(6):39383946. doi: 10.21037/apm-20-2083 